\title{
Ce-Doped CuMgAl Oxide as a Redox Couple Mediated Catalyst for Visible Light Aided Photooxidation of Organic Pollutants
}

\author{
Karan Goswami ${ }^{\mathrm{a}}$ and Rajakumar Ananthakrishnan ${ }^{\mathrm{a} *}$ \\ a Department of Chemistry, Green Environmental Materials \& Analytical Chemistry \\ Laboratory, Indian Institute of Technology Kharagpur, Kharagpur 721302, India. \\ *E-mail: raja@chem.iitkgp.ac.in
}

\section{FT-IR Spectra of the MMOs}

Fourier transform infrared spectroscopy was recorded by Perkin Elmer FT-IR Spectrophotometer RXI.

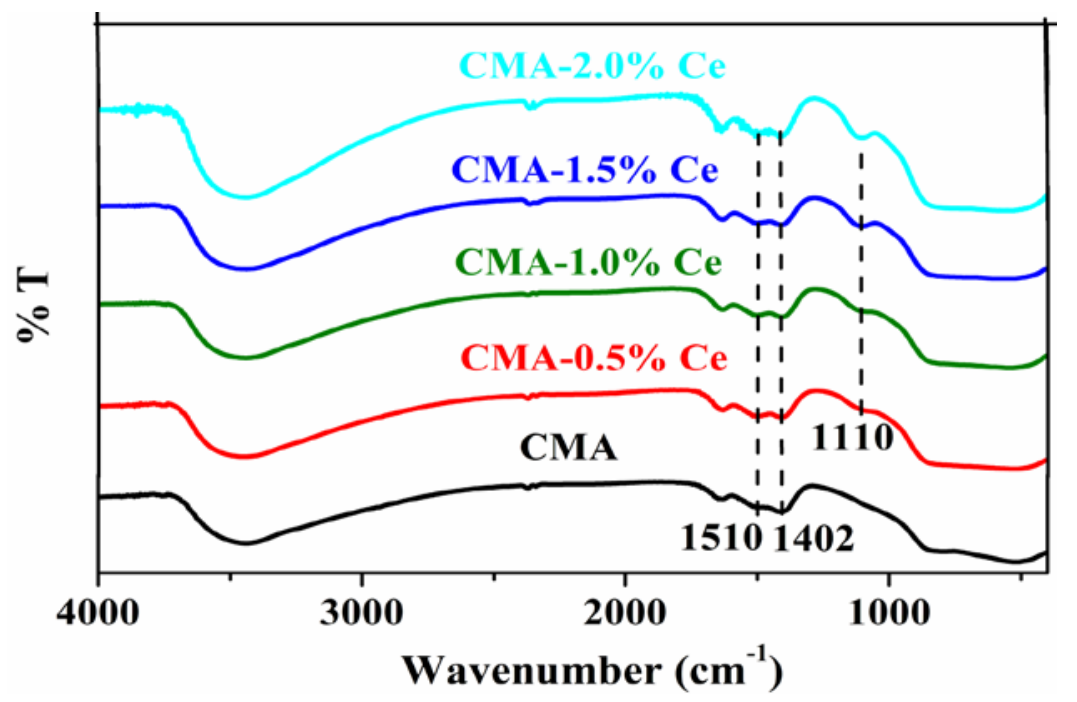

Figure S1. FT-IR spectra of prepared MMO samples.

All the samples have shown two broad peaks at around $3450 \mathrm{~cm}^{-1}$ and $1640 \mathrm{~cm}^{-1}$ due stretching and bending frequencies of $\mathrm{OH}$ group respectively. The bands in the ranges of 400 $\mathrm{cm}^{-1}$ to $800 \mathrm{~cm}^{-1}$ are related to the characteristic vibrational frequencies of M-O bond. X. Jiao et al. ${ }^{1}$ had shown that FT-IR spectrum of MgAl-MMO generally shows two significant peaks at $1402 \mathrm{~cm}^{-1}$ due to asymmetric stretching vibration of unidentate $\mathrm{MgO}$ and $1510 \mathrm{~cm}^{-1}$ due to symmetric stretching vibration of bidentate species. In addition, one new peak at around 1110 $\mathrm{cm}^{-1}$ was observed in Ce doped CuMgAl-MMOs which may be attributed to the stretching frequency of Ce-O bond. 

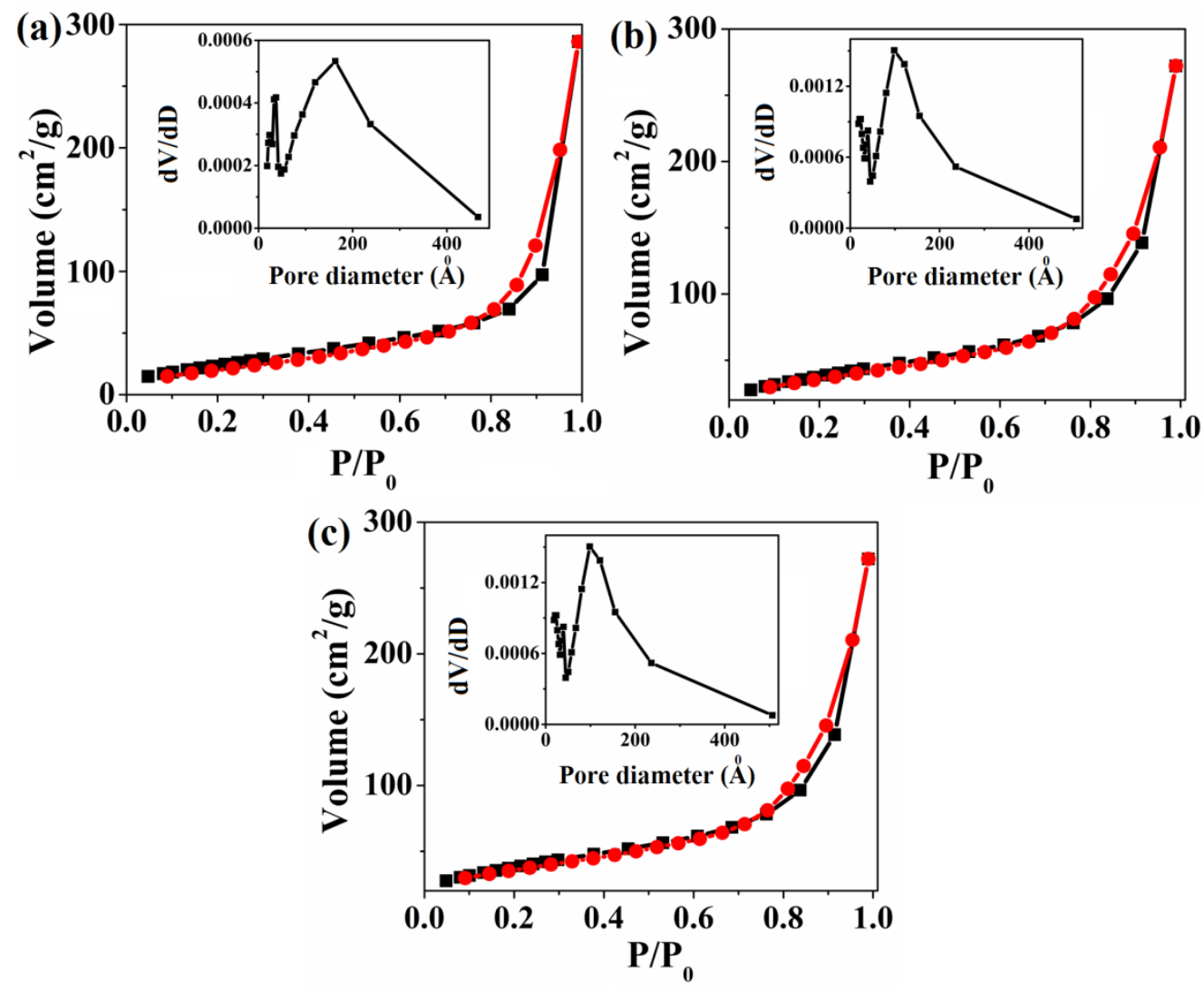

Figure S2. $\mathrm{N}_{2}$ adsorption-desorption isotherms and corresponding pore size distributions (inset) of materials: (a) CMA-0.5\% Ce (b) CMA-1.5\% Ce and (c) CMA-2.0\% Ce.

(a)

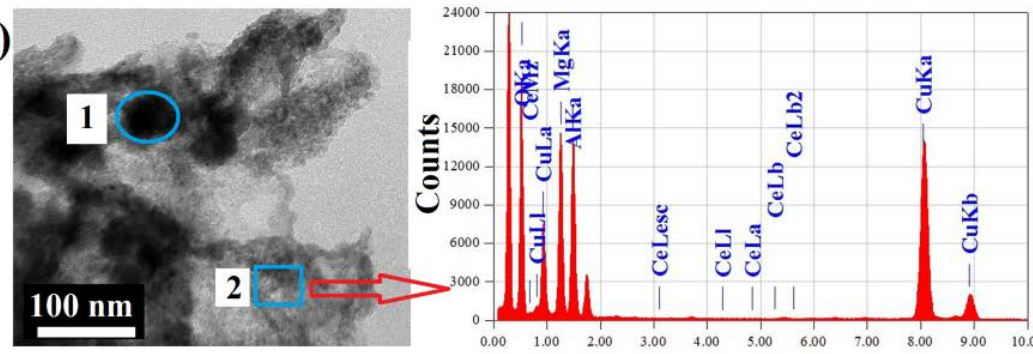

\begin{tabular}{|lcc|}
\hline Element & Mass\% & Atom\% \\
O K & 25 & 46 \\
Mg K & 13 & 16 \\
Al K & 13 & 15 \\
Cu K (Ref.) & 49 & 23 \\
Ce L & ND & ND \\
Total & 100.00 & 100.00 \\
\hline
\end{tabular}

(b)

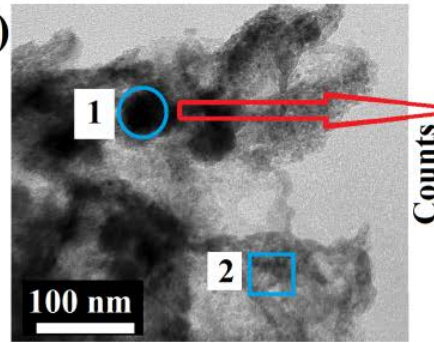

Energy $(\mathrm{KeV})$

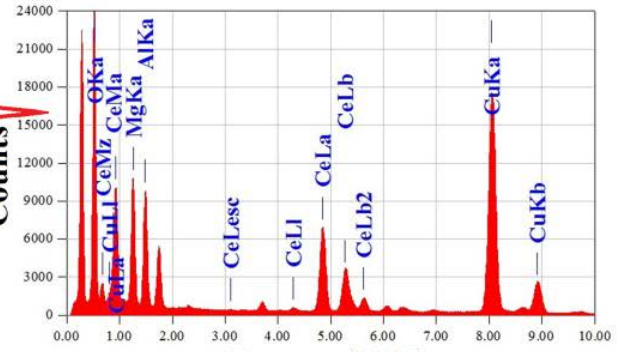

\begin{tabular}{|lcc|}
\hline Element & Mass\% & Atom\% \\
O K & 20 & 50 \\
Mg K & 6 & 9 \\
Al K & 6 & 8 \\
Cu K (Ref.) & 41 & 25 \\
Ce L & 27 & 8 \\
Total & 100.00 & 100.00 \\
\hline
\end{tabular}

Energy (KeV)

Figure S3. EDX analysis of CMA-2.0\% Ce recorded at (a) position '2', and (b) position ' 1 '. 


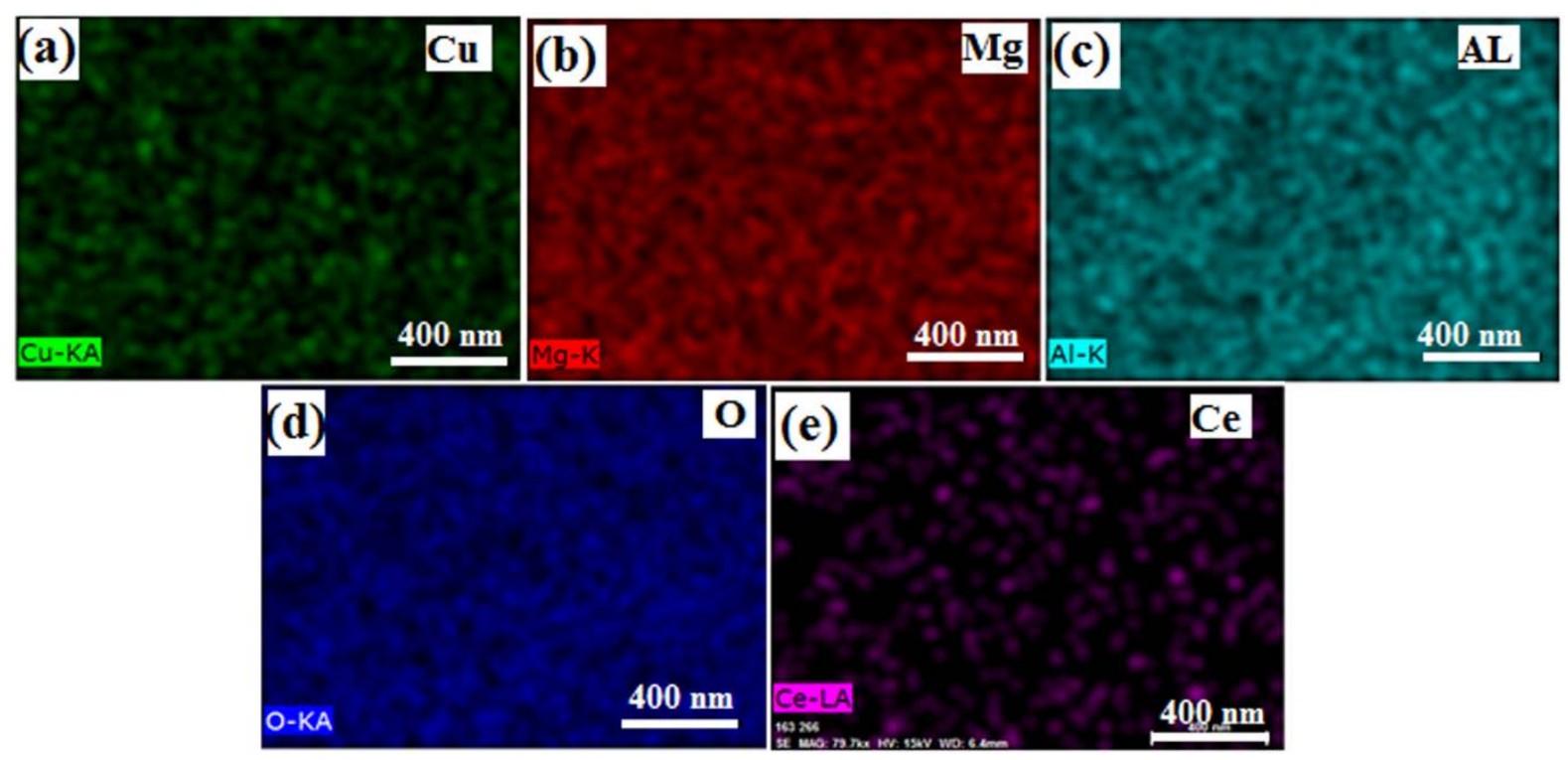

Figure S4. EDX mapping of the elements present in CMA-2.0\% Ce analyzed from FESEM. 


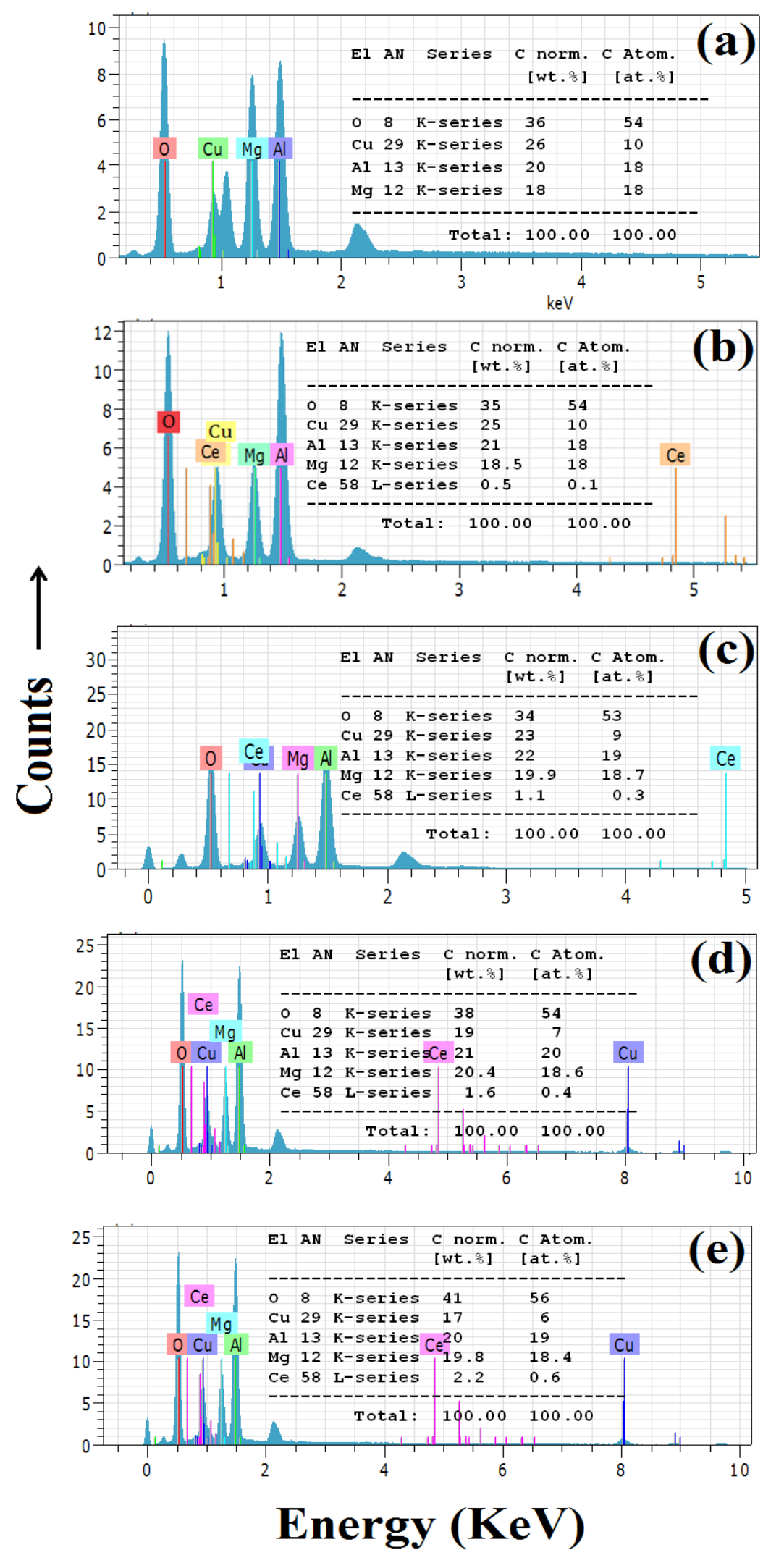

Figure S5. EDX analysis of (a) CMA, (b) CMA-0.5\% Ce, (c) CMA-1.0\% Ce, (d) CMA1.5\% Ce and (e) CMA-2.0\% Ce. 

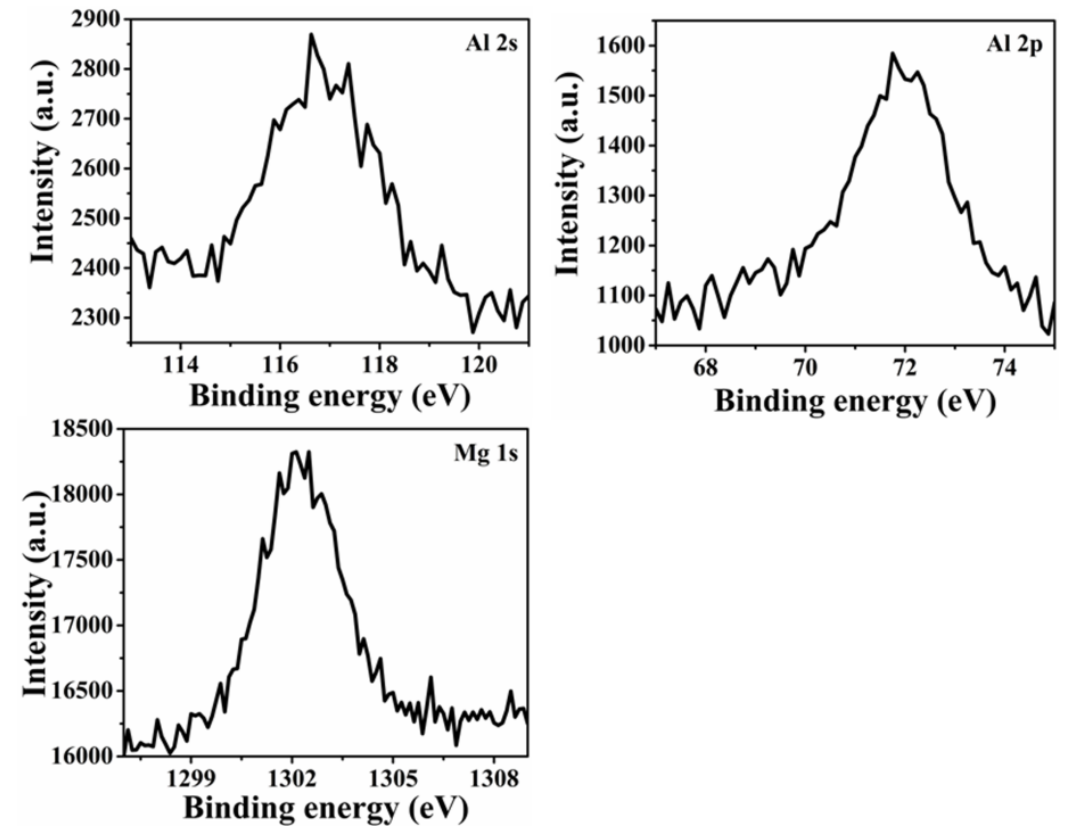

Figure S6. XPS spectra of CMA-1.0\% Ce showing energy levels of Al 2s, Al-2p, and Mg-1s.


Figure S7. Tauc plot of (a) CMA-1.0\% Ce and (b) CMA-1.5\% Ce. 

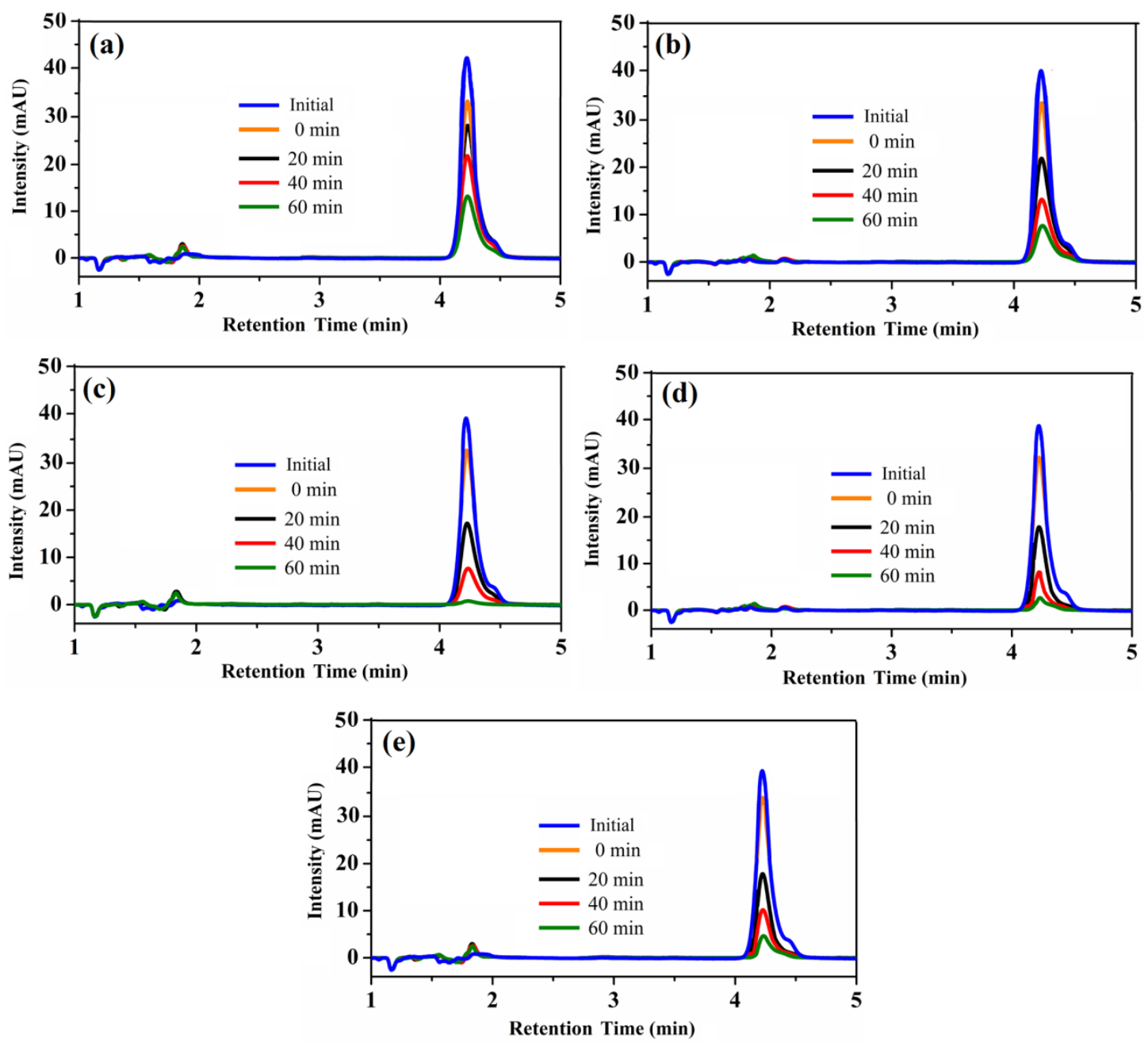

Figure S8. HPLC chromatograms of 2,4-DCP after photodegradation under visible light by: (a) CMA, (b) CMA-0.5\% Ce, (c) CMA-1.0\% Ce, (d) CMA-1.5\% Ce and (e) CMA-2.0\% Ce.

Photoelectrochemical Measurements: The photoelectrochemical (PEC) measurements were conducted in a conventional three electrode system using a CHI 760D electrochemical workstation (CH Instruments, Inc., USA). The three electrode system was composed of a catalyst coated indium-doped tin oxide (FTO) glass $(1 \mathrm{~cm} \times 1 \mathrm{~cm})$ as working electrode, a Pt wire as counter electrode, and a saturated calomel electrode (SCE) as reference electrode. The electrochemical studies were carried out in $0.5 \mathrm{M} \mathrm{Na}{ }_{2} \mathrm{SO}_{4}$ electrolyte at room temperature. The working electrode was prepared by drop casting the as-prepared active material slurry on FTO glass substrate. The slurry was prepared by mixing $5 \mathrm{mg}$ of the assynthesized powder sample in $0.5 \mathrm{~mL}$ of NMP, followed by addition of PVDF (9:1) as binder. The linear sweep voltammetric measurement was performed in $-0.8 \mathrm{~V}$ to $0.2 \mathrm{~V}$ potential window with $20 \mathrm{mV} / \mathrm{s}$ scan rate under irradiation $300 \mathrm{~W}$ xenon lamp on-off condition. The transient photocurrent $(\mathrm{i}-\mathrm{t})$ measurement was performed at an applied potential of $-0.4 \mathrm{~V}$ under chopped light irradiation (light on/off) using the same $300 \mathrm{~W}$ xenon 
lamp. The electrochemical impedance spectroscopy (EIS) study was performed in the frequency range of $100 \mathrm{mHz}$ to $100 \mathrm{kHz}$ with a sinusoidal AC signal of $10 \mathrm{mV}$ at a bias potential of $0 \mathrm{~V}$ (versus SCE) in $0.5 \mathrm{M} \mathrm{Na}_{2} \mathrm{SO}_{4}$ electrolyte.

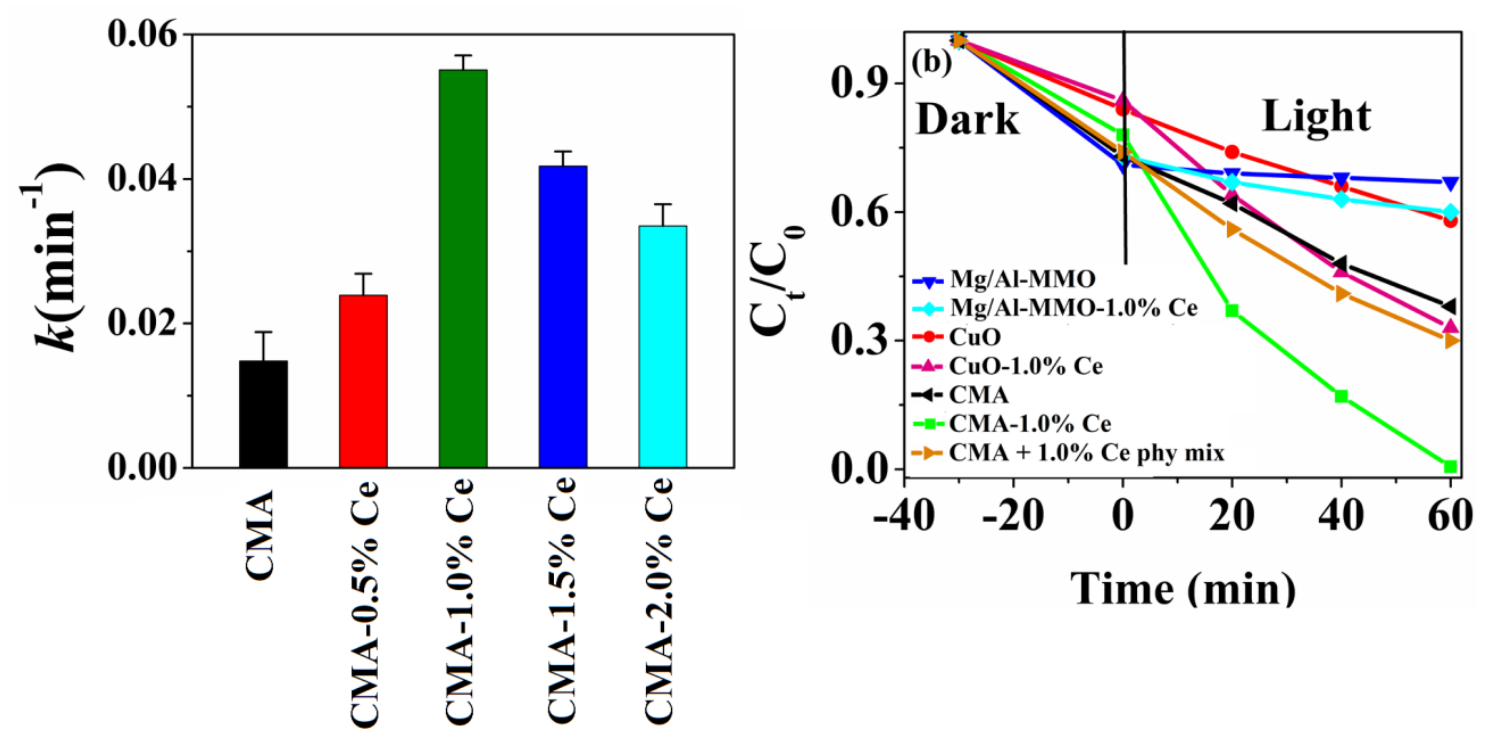

Figure S9. (a) Rate constant values for degradation of 2,4-DCP by different. (b) Comparison of photocatalytic activity of various samples on the degradation of 2,4-DCP under the visible light.

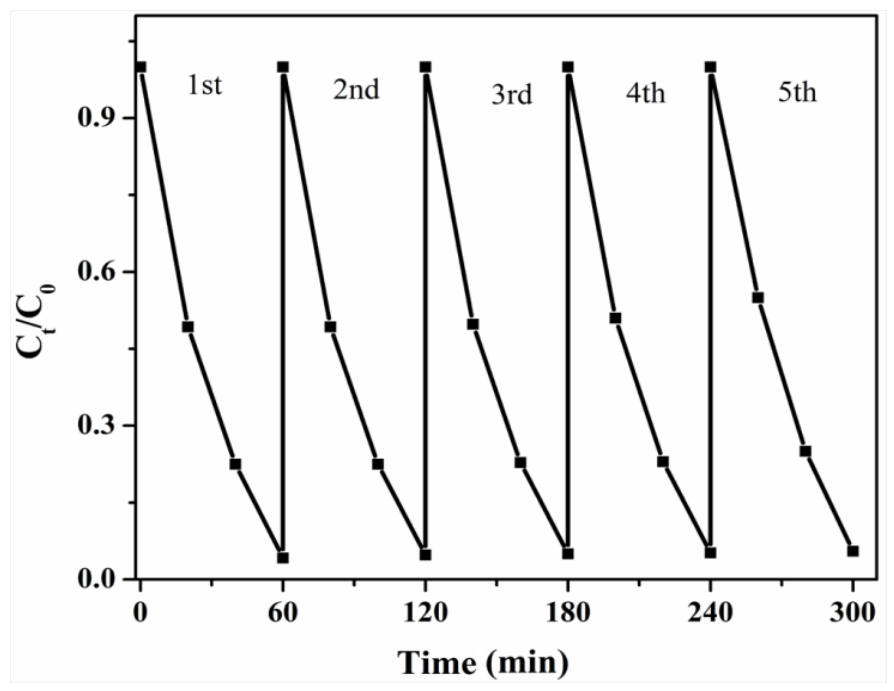

Figure S10. Reusability studies of CMA-1.0\% Ce and its activities upto 5 consecutive cycles. 

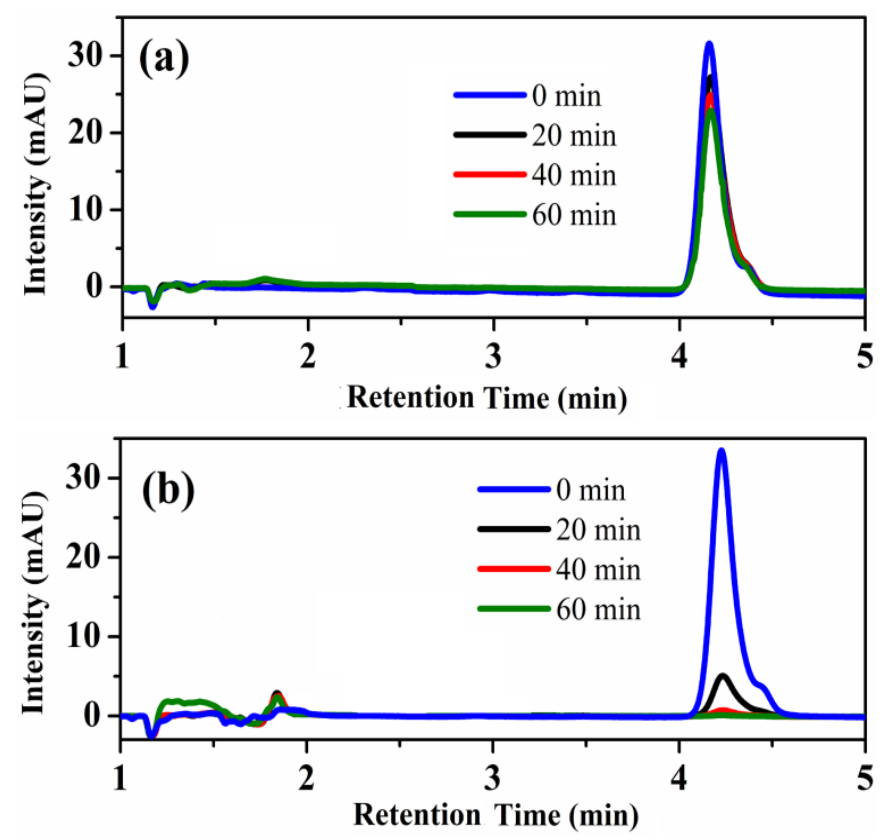

Figure S11. HPLC chromatograms of degradation of 2,4-DCP over CMA-1.0\% Ce under (a) UV light irradiation, and (b) UV-vis light irradiation.

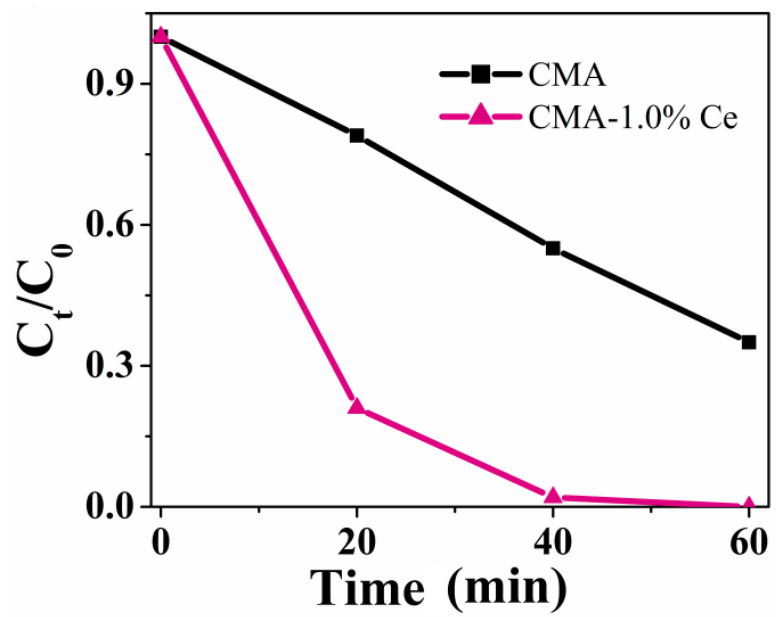

Figure S12. Comparison of photocatalytic activity of CMA and CMA-1.0\% Ce on the degradation of 2,4-DCP under the UV-vis light irradiation. 




Figure S13. UV-vis spectra for degradation of MB dye by CMA-1.0\% Ce under the visible light. Experimental conditions: $[\mathrm{MB}]=10 \mathrm{ppm}, \mathrm{CMA}-1.0 \% \mathrm{Ce}=1.0 \mathrm{~g} / \mathrm{L}$, and source of light $=250 \mathrm{~W}$ tungsten lamp.

\section{Experimental Procedure for Degradation of MB dye:}

Exactly, $20 \mathrm{~mL}$ aqueous solution of the contaminants $(10 \mathrm{ppm})$ and catalyst $(1 \mathrm{~g} / \mathrm{L})$ were taken in a $30 \mathrm{~mL}$ of quartz vial and was dispersed by ultrasonication for $10 \mathrm{~min}$. The suspension was vigorously agitated for $30 \mathrm{~min}$ in the dark to allow adsorption of the contaminants on the catalyst surface before irradiation. About $3 \mathrm{~mL}$ of suspension was collected and centrifuged to remove the solid particle and denoted as initial concentration for photocatalysis. Then, the remaining solution was irradiated under visible light (250 W tungsten lamp). At the given time interval of $15 \mathrm{~min}, 3 \mathrm{~mL}$ of suspension was collected and centrifuged and the concentration of the supernatant was determined using UV-1601 SHIMADZU spectrophotometer.

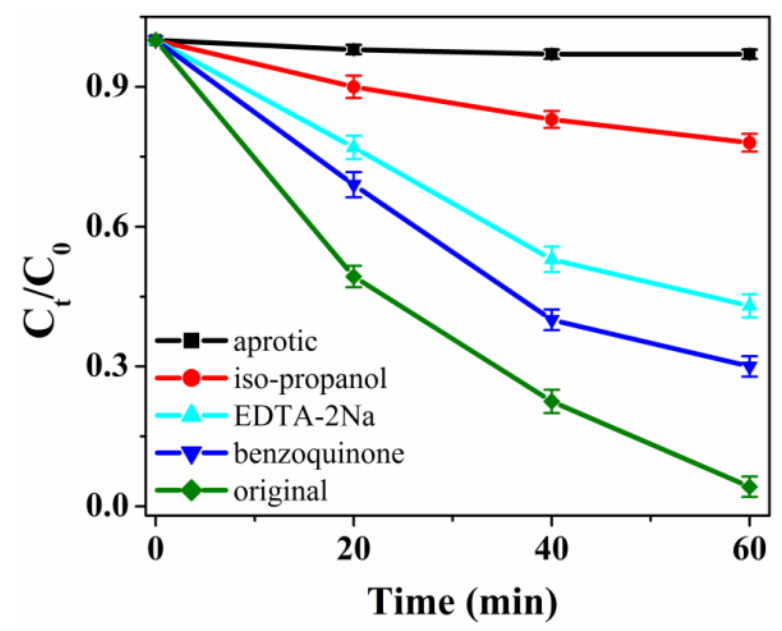

Figure S14. Control experiment with scavengers of photogenerated active species; here, EDTA-2Na as $\mathrm{h}^{+}$scavenger, 2-propanol as $\bullet \mathrm{OH}$ scavenger and benzoquinone as $\cdot \mathrm{O}_{2}{ }^{-}$ scavenger. 
(a)

$\mathrm{HOOC}_{\smile} \mathrm{COOH}$

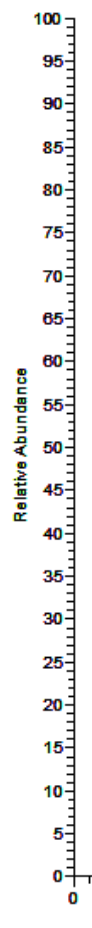

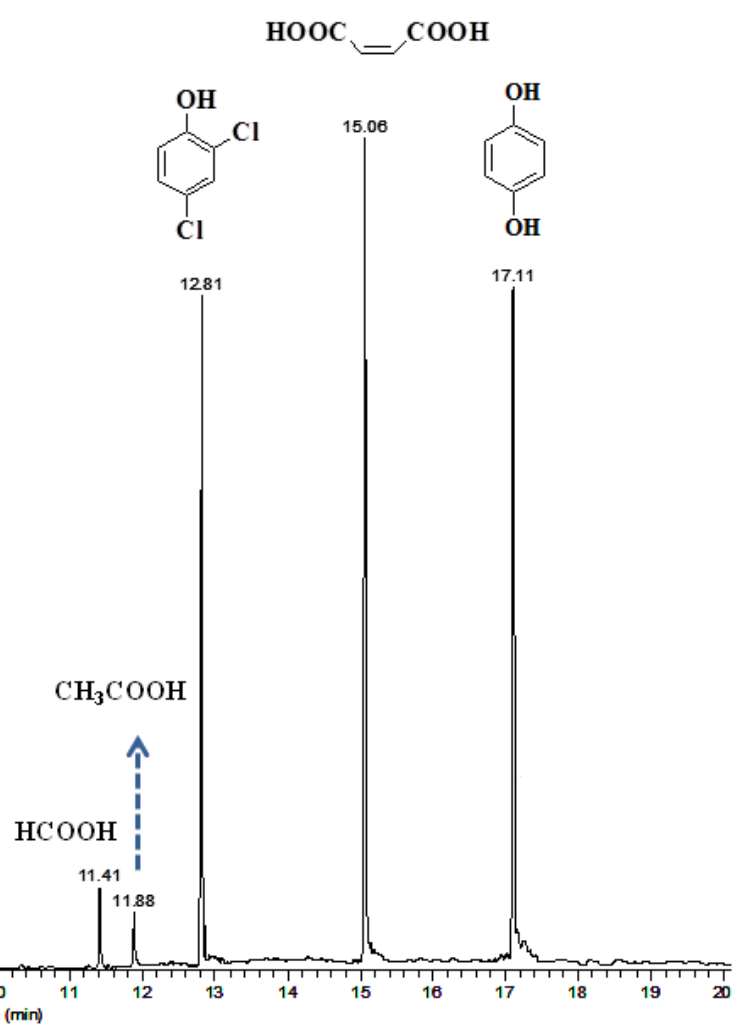

(b)

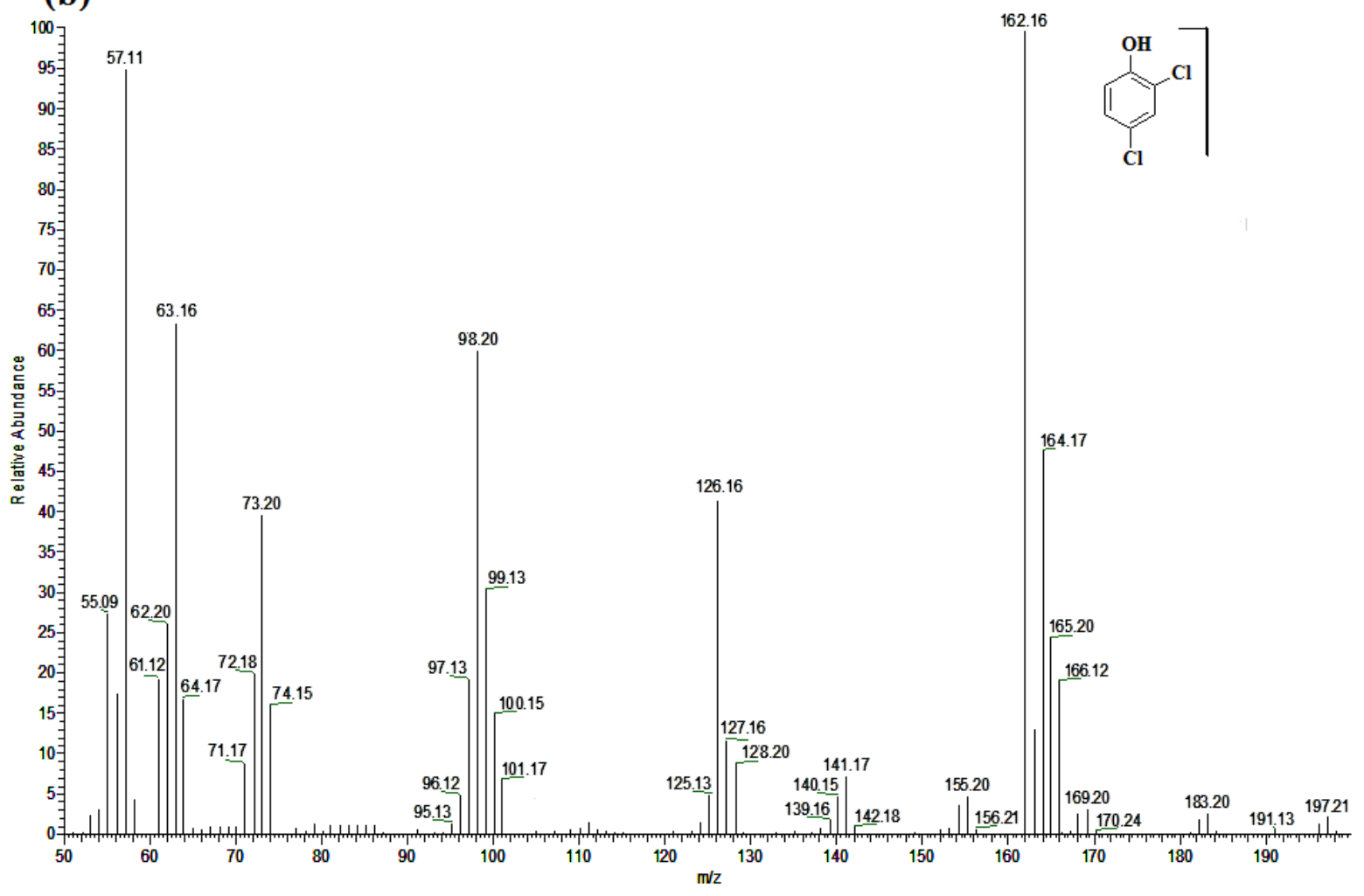

Continued..... 
(c)

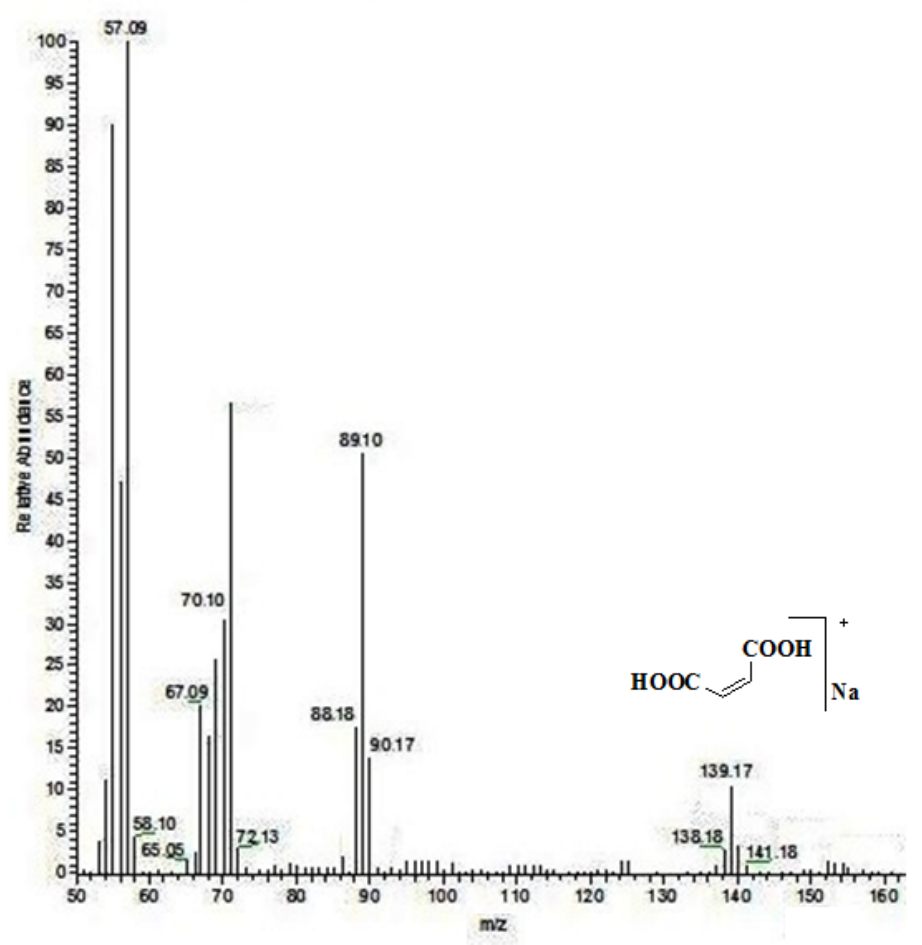

(d)

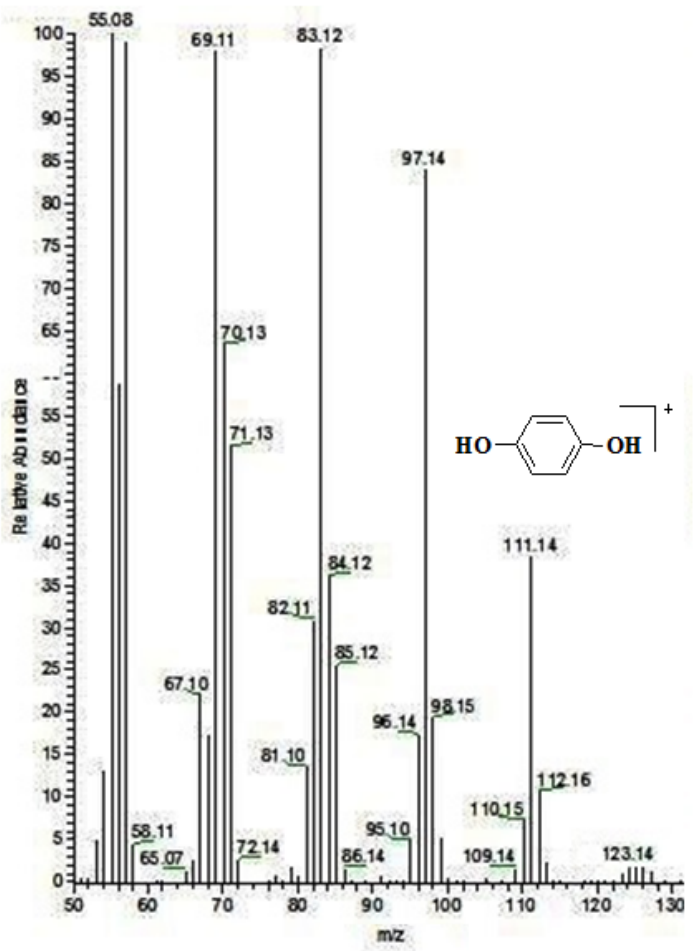

Figure S15. (a) Gas chromatogram (GC) of 2,4-DCP after 20 min of photodegradation by the catalyst, and (b)-(d) are the corresponding mass spectra of the GC chromatogram.

Table S1. Summary of Lattice Parameters and Crystallite Size Values of LDH Samples.

\begin{tabular}{llll}
\hline \multirow{2}{*}{ samples } & \multicolumn{2}{c}{ lattice parameters (nm) } & crystallite \\
\cline { 2 - 3 } & $\mathrm{a}=2 \mathrm{~d} 110$ & $\mathrm{c}=3 \mathrm{~d} 003$ & \\
\hline CMAe $(\mathrm{nm})$
\end{tabular}


Table S2. Comparison of Photocatalytic Activity of the Prepared Material with Reported Materials in Literature.

\begin{tabular}{|c|c|c|c|c|}
\hline materials & experimental conditions & $\begin{array}{l}\text { degra- } \\
\text { dation } \\
(\%)\end{array}$ & $\begin{array}{l}\text { rate } \\
\text { constant } \\
k\left(\mathrm{~min}^{-1}\right)\end{array}$ & reference \\
\hline \multicolumn{5}{|c|}{ A. Comparison of 2,4-DCP degradation by different materials and CMA-1.0\% Ce catalyst: } \\
\hline 1. $\mathrm{CuO}_{\mathrm{x}} / \alpha-\mathrm{Bi}_{2} \mathrm{O}_{3}$ & $\begin{array}{l}\text { 2,4-DCP conc. }=\sim 10 \mathrm{ppm} \\
\text { Cat. }=1 \mathrm{~g} / \mathrm{L} \\
\text { Light }=300 \mathrm{~W} \text { Xe (Vis.) } \\
\text { Irradiation time }=420 \mathrm{~min} .\end{array}$ & $\sim 90$ & - & $\begin{array}{l}\text { Jiang. et al. } \\
2015^{2}\end{array}$ \\
\hline 2. $0.075 w t \% P t / \mathrm{Bi}_{2} \mathrm{O}_{3}$ & $\begin{array}{l}\text { 2,4-DCP conc. }=\sim 10 \mathrm{ppm} \\
\text { Cat. }=1 \mathrm{~g} / \mathrm{L} \\
\text { Light }=300 \mathrm{~W} \text { Xe (Vis.) } \\
\text { Irradiation time = } 240 \mathrm{~min} .\end{array}$ & $\sim 60$ & - & $\begin{array}{l}\text { Yang. et al. } \\
2017^{3}\end{array}$ \\
\hline 3. CdS@ $\mathrm{TiO}_{2}$ & $\begin{array}{l}\text { 2,4-DCP conc. = } 10 \mathrm{ppm} \\
\text { Cat. = } 1.0 \mathrm{~g} / \mathrm{L} \\
\text { Light = Mic-LED-455 (Vis.) } \\
\text { Irradiation time = } 360 \mathrm{~min} .\end{array}$ & $\sim 74$ & 0.0037 & $\begin{array}{l}\text { Al-Fahdi. } \\
\text { et al. } 2019^{4}\end{array}$ \\
\hline 4. 2\% GO-Polyimide & $\begin{array}{l}\text { 2,4-DCP conc.= } 10 \mathrm{ppm} \\
\text { Cat. }=10 \mathrm{mg} / \mathrm{L} \\
\text { Light }=300 \mathrm{~W} \text { Xe (Vis.) } \\
\text { Irradiation time = } 120 \mathrm{~min} .\end{array}$ & $\sim 95$ & 0.0242 & $\begin{array}{l}\text { Gong. et al. } \\
2019^{5}\end{array}$ \\
\hline \multirow[t]{3}{*}{ 5. CMA-1.0\% Ce } & $\begin{array}{l}\text { 2,4-DCP conc. }=10 \mathrm{ppm} \\
\text { Cat. }=1 \mathrm{~g} / \mathrm{L}\end{array}$ & & & \\
\hline & $\begin{array}{l}\text { Light }=250 \mathrm{~W} \text { tungsten (Vis.) } \\
\text { Irradiation time }=60 \mathrm{~min} .\end{array}$ & 96 & 0.0551 & This work \\
\hline & $\begin{array}{l}250 \mathrm{~W} \text { (UV and Vis.) } \\
\text { Irradiation time }=45 \mathrm{~min} .\end{array}$ & 100 & 0.0796 & \\
\hline
\end{tabular}

B. Comparison of MB degradation by different Cu-Ce based catalysts and CMA-1.0\% Ce catalyst:

\begin{tabular}{|c|c|c|c|c|}
\hline 6. $\mathrm{Cu}$ doped $\mathrm{CeO}_{2}$ & $\begin{array}{l}\text { MB dye conc. }=15 \text { ppm; Cat. }=1 \mathrm{~g} / \mathrm{L} \\
\text { Light }=\text { Xenon lamp }(\lambda>400 \mathrm{~nm}, 635 \mathrm{~lx}) \\
\text { Irradiation time }=300 \mathrm{~min}\end{array}$ & 97.83 & 0.0143 & $\begin{array}{l}\text { Ranjith. } \\
\text { et al. } 2018^{6}\end{array}$ \\
\hline 7. Ce doped $\mathrm{CuO}$ & $\begin{array}{l}\text { MB dye conc. }=10^{-5} \mathrm{M} \text {; Cat. }=5 \mathrm{~g} / \mathrm{L} \\
\text { Light }=\mathrm{UV} \text { lamp } \\
\text { Irradiation time }=180 \mathrm{~min}\end{array}$ & 31 & - & $\begin{array}{l}\text { Ekthammat } \\
\text { hat. et al. } \\
2016^{7}\end{array}$ \\
\hline 8. CMA-1.0\% Ce & $\begin{array}{l}\text { MB dye conc. = } 10 \mathrm{ppm} \text {; Cat. = } 1 \mathrm{~g} / \mathrm{L} \\
\text { Light }=250 \mathrm{~W} \text { tungsten (vis.) } \\
\text { Irradiation time }=90 \mathrm{~min}\end{array}$ & 100 & 0.0362 & This work \\
\hline
\end{tabular}


1. Jiao, X.; Li, H.; Li, L.; Xiao, F.; Zhao, N.; Wei, W. Synthesis and $\mathrm{CO}_{2}$ Capture Properties of Mesoporous MgAl(O) Sorbent. RSC Adv. 2014, 4, 47012-47020.

2. Jiang, H.-Y.; Liu, G.; Li, M.; Liu, J.; Sun, W.; Ye, J.; Lin, J. Efficient Organic Degradation under Visible Light by $\alpha-\mathrm{Bi}_{2} \mathrm{O}_{3}$ with a $\mathrm{CuO}_{\mathrm{x}}$-Assistant Electron Transfer Process. Appl. Catal. B: Environ. 2015, 163, 267-276.

3. Yang, K.; Li, J.; Peng, Y.; Lin, J. Enhanced Visible Light Photocatalysis over Pt-Loaded $\mathrm{Bi}_{2} \mathrm{O}_{3}$ : An Insight into its Photogenerated Charge Separation, Transfer and Capture. Phys. Chem. Chem. Phys. 2017, 19, 251-257.

4. Al-Fahdi, T.; Al Marzouqi, F.; Kuvarega, A. T.; Mamba, B. B.; Al Kindy, S. M. Z.; Kim, Y.; Selvaraj, R. Visible Light Active CdS@ $\mathrm{TiO}_{2}$ Core-Shell Nanostructures for the Photodegradation of Chlorophenols. J. Photochem. Photobio. A: Chem. 2019, 374, 75-83.

5. Gong, Y.; Yang, B.; Zhang, H.; Zhao, X.; Zhu, C. Graphene Oxide Enwrapped Polyimide Composites with Efficient Photocatalytic Activity for 2,4-Dichlorophenol Degradation under Visible Light Irradiation. Mater. Res. Bull. 2019, 112, 115-123.

6. Ranjith, K. S.; Dong, C-L.; Lu, Y-R.; Huang, Y-C.; Chen, C-L.; Saravanan, P.; Asokan, K.; Kumar, R. T. R. Evolution of Visible Photocatalytic Properties of Cu-Doped $\mathrm{CeO}_{2}$ Nanoparticles: Role of $\mathrm{Cu}^{2+-}$ Mediated Oxygen Vacancies and the Mixed-Valence States of Ce Ions. ACS Sustain. Chem. Eng. 2018, 6, 8536-8546.

7. Ekthammathat, N.; Phuruangrat, A.; Thongtem, T.; Thongtem, S. Synthesis and Characterization of Ce-doped CuO Nanostructures and Their Photocatalytic Activities. Mater. Lett. 2016, 167, 266-269. 